\title{
Vorwort zur 39. Auflage
}

Die Überarbeitung und Aktualisierung der 39. Auflage ist geprägt durch das Gesetz zum Bürokratieabbau und zur Förderung der Transparenz bei Genossenschaften (BGBl. I 2017, S. 2434). Bei der GenG-Novelle 2017 handelt es sich um die umfangreichsten Änderungen des Genossenschaftsgesetzes seit der GenG-Novelle 2006 (BGBl. I 2006, S. 2230); schon im Hinblick darauf war eine Überarbeitung des Kommentars geboten.

In diesem Zusammenhang ist die vollständige Neukommentierung der Vorschrift zu den Mitgliederdarlehen ( $§ 21 \mathrm{~b}$ GenG) und zur vereinfachten Prüfung ( 5 53a GenG) hervorzuheben. Erste Auslegungs- und Anwendungsfragen im Zusammenhang mit den neuen Vorschriften, die sich in der genossenschaftlichen Prüfung ergaben, konnten bereits praxisbezogen beantwortet werden.

Die Änderungen durch das Abschlussprüfungsreformgesetz (AReG) und Abschlussprüferaufsichtsreformgesetz (APAReG) waren schon in der Fassung des Gesetzesentwurfs in der 38. Auflage berücksichtigt und sind jetzt in deren Endfassung eingearbeitet worden. Ebenso sind die weiteren Änderungen durch das CSR-Richtlinie-Umsetzungsgesetz (BGBl. I 2017, S. 802) in § 38 GenG, Zweites Finanzmarktnovellierungsgesetz (BGBl. I 2017, S. 1693) in $\S 166$ GenG und das Gesetz zur Änderung des Bundesversorgungsgesetzes und anderer Vorschriften (BGBl. I 2017, S. 2541) in §§ 31, 156 GenG berücksichtigt worden. Das Bundesdatenschutzgesetz (BDSG) ist im Mai 2018 durch die einheitliche europäische Datenschutz-Grundverordnung (DSG-VO; EU-Abl. L 17 v. 23.5.2018) und ein Datenschutzanpassungs- und -umsetzungsgesetz (BGBl. I 2017, S. 2097) ersetzt worden, die entsprechenden Regelungen und Verweise wurden aktualisiert.

Aufgrund von Anregungen aus der Praxis ist am Ende der Einführung eine Übersicht zur Kommentierung der Vorschriften zur Europäischen Genossenschaft (SCE-Verordnung) aufgenommen worden. Die Übersicht soll, ausgehend vom jeweiligen Artikel der SCE-Verordnung, das Auffinden der Kommentierung bei der entsprechenden Vorschrift im GenG erleichtern.

Darüber hinaus erfolgte eine umfassende Aktualisierung des Kommentars um zwischenzeitlich veröffentlichte Rechtsprechung und Literatur. Der Kommentar ist damit auf dem Stand August 2018.

Bonn, im August 2018

Dirk J. Lehnhoff

Jan Holthaus 
